\title{
Metodologia
}

\author{
Técnicas para \\ obtenção e tratamento de macrofotografias \\ de insetos \\ Techniques for \\ achievement and processing of insect \\ macrophotographs
}

\author{
RicARDO T. FUJIHARA ${ }^{1}$ \\ LuIz C. FORTI ${ }^{1}$
}

As fotografias são um importante meio para transmissão de informações, podendo servir para publicações científicas, conferências e cursos, auxiliando assim na apresentação de resultados com maior riqueza de detalhes (Trevisan et al., 2002; Hochman et al., 2005).

Quando incorporadas a um banco de dados, podem aumentar a habilidade dos parataxonomistas no reconhecimento de morfoespécies, servindo também como ferramentas para ensino, análises preliminares de dados de campo e armazenamento de informação (BASSET et al., 2000).

Além disso, as fotografias são de grande valia na elaboração de chaves de identificação pictóricas, interativas e hipertexto, apresentando imagens anexas que ilustram o caráter taxonômico (SElTMAnN, 2004). Segundo PaPAVERo \& Martins (1994), estas facilitam o uso das chaves e provavelmente resultam em um maior número de identificações corretas.

\footnotetext{
${ }^{1}$ Laboratório de Insetos Sociais-Praga, Departamento de Produção Vegetal, Faculdade de Ciências Agronômicas (UNESP) - C. Postal 237, Botucatu SP, Brasil - CEP 18610-307 _ E-mail: rtfujihara@ hotmail.com.

${ }^{2}$ Verdete $($ verdigris $)=$ ácidos orgânicos oriundos do corpo dos insetos que reagem com o cobre, latão ou bronze dos alfinetes entomológicos, formando uma substância de cor esverdeada, o óxido de cobre. Atualmente os alfinetes são fabricados em aço inoxidável, reduzindo e ou eliminando esse problema.
} 
No entanto, técnicas que visam à obtenção e tratamento de macrofotografias de insetos e outros organismos são pouco difundidas no meio científico. A redução nos valores dos equipamentos, como câmeras fotográficas, microcomputadores, impressoras, microscópios e estereoscópios, tem possibilitado implementar novas metodologias e ferramentas, com ótima relação custo-benefício.

Assim, por meio de conhecimentos próprios e/ou adquiridos por especialistas, foram desenvolvidas metodologias para a aquisição e otimização de macrofotografias em alta definição (resolução), visando melhorar sua qualidade, sem alterar, no entanto, suas características técnicas. No curso de nossas atividades em nosso laboratório enfrentamos a necessidade de desenvolver aspectos que aprimorassem esse trabalho. Com intuito de contribuir com o trabalho ligado a ilustração de publicaçãos, apresentamos abaixo a nossa experiência nesse aspecto.

\section{PREPARAÇÃODOS ESPÉCIMES}

Primeiramente, os espécimes foram submetidos a um tratamento para limpeza e remoção de partículas (poeira, fungos, verdete ${ }^{2}$ ) impregnadas no tegumento, melhorando seu aspecto visual para as fotografias.

Considerando a conservação em via seca, é recomendável que todos os insetos passem por este processo, excetuando-se alguns grupos. A utilização de compostos abrasivos ocasiona danos nas asas grandes e frágeis de Lepidotera, Trichoptera, Neuroptera e Odonata, e insetos delicados ou de tamanho reduzido, como os dípteros da família Culicidae e microhimenópteros, que podem ter parte de suas estruturas danificadas.

A área afetada pelo verdete pode ser removida com acetona ou éter de petróleo. Pincelar de modo que a solução penetre no interior do espécime, removendo cuidadosamente em seguida com o pincel. O verdete irá retornar gradativamente após um ou dois anos, devendo-se repetir o processo quando necessário. Para a retirada das demais partículas, pode ser utilizado fosfato trissódico $\left(\mathrm{Ia}_{3} \mathrm{NO}_{4}\right)$ a $1 \%$ em água fria. Os insetos devem ser "mergulhados" nessa solução contendo uma gota de detergente neutro por 24 horas, lavando-os em seguida com etanol (WALKer \& CROSby, 1988).

Segundo Azevedo Filho et al. (2007), outra alternativa seria o uso de álcool isopropílico ou isopropanol pincelado diretamente sobre os espécimes.

No entanto, com fins facilitadores, utilizamos éter etílico PA (próanálise) a 70\%. Os insetos foram "mergulhados" por cerca de 30 segundos em um béquer contendo a solução. Em seguida, com o auxílio de um pincel (marca Tigre ${ }^{\circledR}$, número 266) foram retiradas eventuais partículas ainda impregnadas no tegumento. 
Cuidado redobrado ao manusear tais substâncias, pois além de inflamáveis, podem causar intoxicação, irritação e queimaduras sob exposição prolongada.

\section{OBTENÇÃO DE IMAGENS COM CÂMERA DIGITAL SLR (SINGLELENS REFLEX)}

Caso tenha dificuldade em interpretar os termos técnicos fotográficos, o website Photonotes.org (http://photonotes.org/dictionary) disponibiliza um dicionário com mais de 1.480 definições. Outros, como Fujifilm Brasil $^{\circledast}$ (http://www.fujifilm.com.br/comunidade/dicas/index.html) e $\operatorname{Kodak}^{\circledR}$ (http://wwwbr.kodak.com/BR/pt/consumer/fotografia _digital _classica/para_uma_boa_foto/curso_fotografia/curso_ fotografia. shtml?primeiro=1) apresentam dicas e informações sobre fotografia tradicional e digital.

Inicialmente foi construído um mini-estúdio composto por três luminárias alógenas de 150 watts/bivolts e duas lâmpadas de mercúrio mistas de 250 watts/220 volts (Figs 1-A e B). Esta estrutura foi desenvolvida com o intuito de melhorar o nível de iluminação do ambiente, possibilitando a aquisição de macrofotografias de melhor qualidade, nitidez e ausente de sombras, e consequentemente, facilitando no tratamento posterior das mesmas.

Para a obtenção das imagens foi utilizada uma câmera digital Canon ${ }^{\grave{ }}$, modelo EOS Rebel XT, com resolução de 8.0 megapixels (Fig. 1-F). Em conjunto foram utilizados dois modelos de lentes: lente macro Canon ${ }^{\grave{O}}$, modelo MP-E 65 mm 1:2,8 e lente macro Canon ${ }^{\mathrm{E}} \mathrm{EF} 50 \mathrm{~mm}$ 1:2,5. Para insetos transfixados, lentes macro com distância focal entre 50 a $90 \mathrm{~mm}$ são suficientes, pois não é necessária uma distância limite de um objeto inanimado.

Complementando o mini-estúdio foram conectados três flashes Tron ${ }^{o ̀}$ S-300S, um flash Tron ${ }^{\grave{~}} 100$ S e um "ring-flash" STARblitz 1000 Auto Macro-lite (Figs 1-C, D e E). Todos os flashes eram acionados (disparados) simultaneamente por meio de fotocélulas Monte Cristo ${ }^{\mathrm{O}}$, modelo MC 9000, evitando distorções de luminosidade e sombras.

As etiquetas foram cuidadosamente retiradas dos alfinetes entomológicos com o auxílio de uma pinça, sendo recolocadas após a obtenção das imagens. Não se esquecendo que para coleções as etiquetas são imprescindíveis e devem ser fotografadas em conjunto com os espécimes. Os insetos foram fixados por meio do alfinete em um tubo plástico preenchido com massa de modelar escolar, sendo este acoplado a uma placa de isopor recoberta por papel camurça (Figura 1-G). Este suporte elevou a altura dos insetos perante a superfície, eliminando a formação de eventuais sombras nas imagens, que poderiam dificultar no 
posterior tratamento das mesmas. Além disso, foi adicionado próximo a este outro tubo com uma escala afixada (Figura 1-G), a fim de evitar sub ou superdimensionamento e equívocos taxonômicos.

Após testes com vários materiais e tonalidades, optou-se pela utilização de tecido e papel camurça na cor azul, que aumenta os níveis de contraste e reduz os reflexos produzidos pela incidência de luz. Além disso, esta realça os detalhes dos espécimes e seus caracteres morfológicos, melhorando o aspecto estético e facilitando o tratamento das imagens. Segundo alguns autores (Ashworth \& Fogarty, 2003; Seltmann, 2004), o cartão cinza (14 a 18\%) também pode ser utilizado como fundo (background), sendo útil na manutenção da cor dos espécimes, redução dos reflexos da superfície e na calibração do balanço de branco.

Muitos websites ensinam como confeccionar cartões a baixo custo e com similar funcionalidade. Para fixar os insetos, pode ser utilizada massa de modelar de cor semelhante.

Trataremos aqui dos ajustes necessários para a câmera Canon, e que
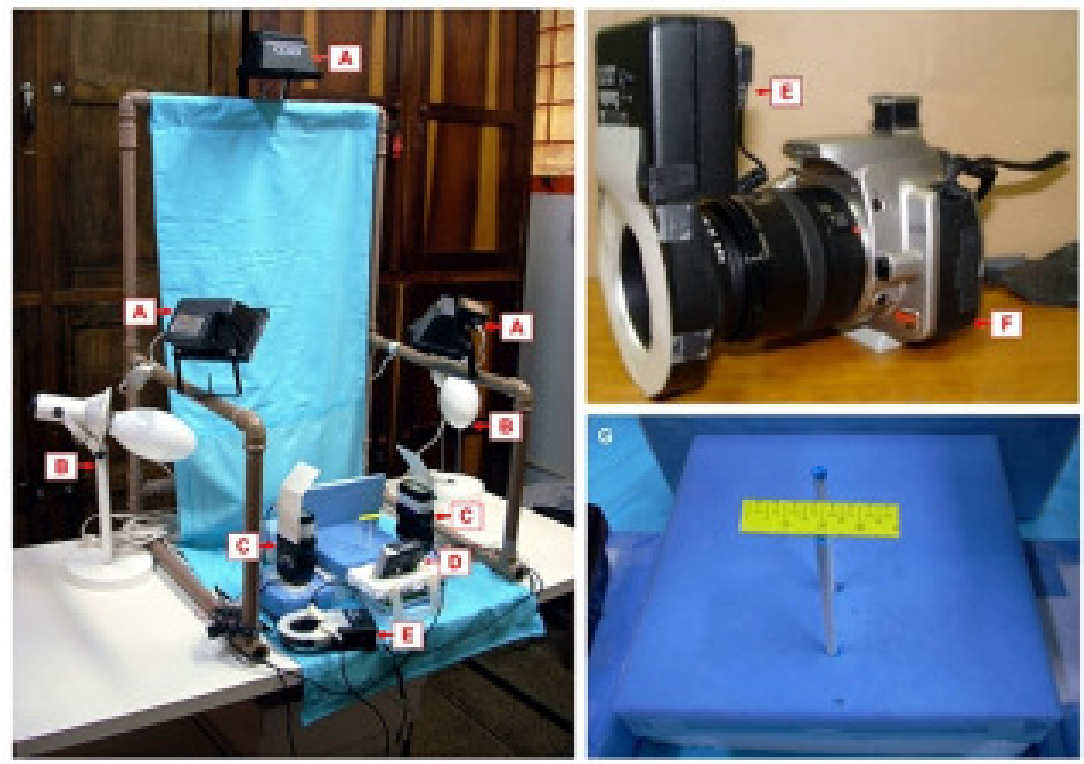

Fig. 1. Estúdio. A. Luminárias alógenas de 150 watts. B. Lâmpadas de mercúrio mistas de 250 watts. C. Flashes Tron $^{\circ}$ S-300S. D. Flash Tron ${ }^{\grave{ }}$ 100S. E. Ring-flash STARblitz 1000 Auto Macro-lite. F. Câmera digital Canon ò , modelo EOS Rebel XT. G. Placa de isopor com tubo plástico para fixação dos insetos. 
no geral, podem ser adotados para a maioria das câmeras digitais SLR:

1. Modo de exposição (exposure mode): manual M.

2. Abertura (aperture): entre os valores f16 a f25, a fim de maximizar a profundidade de campo. Cabe ressaltar que a abertura deve ser ajustada em função do tamanho, coloração do tegumento e características morfológicas do grupo ou inseto amostrado, podendo variar consideravelmente.

3. Tempo de exposição (exposure time): fixo em 1/250.

4. Balanço de branco (white balance): ajustado para captura de fotografias com flash.

5. ISO (International Standards Organization): altos valores de ISO (400 ou >) são geralmente usados em situações de pouquíssima luz, com tempos de exposição menores, e assim, não obtendo fotos borradas ou tremidas. Em contrapartida, estas apresentarão mais ruído (granulação). Assim, recomenda-se fotografar com luminosidade adequada e baixos valores de ISO (100 a 400).

6. Qualidade da imagem (image size): quanto maior a resolução, melhor a qualidade da foto, porém ocupará mais espaço na memória. Imagens com alta resolução perdem menos qualidade durante o tratamento, contudo, requerem microcomputadores com alto desempenho. A mídia na qual as fotos serão utilizadas também é um fator importante. Caso sejam para impressão (como no caso de periódicos) devem apresentar ao menos 300 dpi, mas para internet, tal condição não é obrigatória.

Para a maioria das ordens foram obtidas imagens em vistas lateral e dorsal, com exceção de Lepidoptera, que por convenção, foi fotografada dorsalmente. Insetos vivos foram mantidos sob refrigeração por aproximadamente 5 a 10 minutos, de modo a reduzir o seu metabolismo, e consequentemente, seus movimentos, facilitando assim na captura das imagens.

\section{OBTENÇÃO DE IMAGENS COM CÂMERA DIGITAL ACOPLADA AO ESTEREOSCÓPIO}

Determinadas macrofotografias não puderam ser obtidas por meio da câmera digital SLR, devido ao tamanho diminuto dos insetos $(<5 \mathrm{~mm})$ ou para adquirir detalhes estruturais. Neste caso, foi utilizada uma câmera digital Nikon ${ }^{\text {, }}$, modelo Coolpix 4500, com resolução de 4.0 megapixels (Figura 2-A), acoplada a um microscópio estereoscópico Nikon ${ }^{\mathrm{o}}$, modelo SMZ 1000 (Figura 2-B). Como sua principal desvantagem, não pode ser conectada diretamente a um microcomputador, o que demanda mais 
tempo entre a captura e a visualização das imagens (AsHWORTH \& FOGARTY, 2003).

Existem diversos modelos disponíveis no mercado, mas qualquer câmera digital compacta pode ser utilizada, desde que acoplada a um adaptador. Um exemplo de dispositivo de fácil confecção e com materiais acessíveis pode ser visto em CAtelli et al. (2008). KINCH (2011) descreve com riqueza de detalhes, aspectos relativos à adaptação de câmeras digitais em microscópios e estereoscópios para diferentes aplicações.

Para difundir o brilho e a luminosidade, um copo plástico descartável branco (300 a $500 \mathrm{ml}$ ) foi recoberto com papel sulfite (apenas na face interna) e colocado entre os dois iluminadores do estereoscópio (Fig. 2C). O copo foi então colocado ao redor do espécime, sendo este fixado em placa de isopor em formato de " $L$ ", que facilita a aquisição de imagens em diferentes posições (KLINGENBERG, $2007^{3}$ ).

Ashworth \& Fogarty (2003) descrevem em detalhes todas as configurações para a câmera digital Nikon ${ }^{\text {, }}$, modelo Coolpix 4500. Trataremos aqui dos ajustes necessários para a captura das imagens, e que no geral, podem ser adotados para a maioria das câmeras digitais compactas, acopladas ou não a um estereoscópio:

1. Modo de exposição (exposure mode): manual $\mathbf{M}$.

2. Modo de cena (scene mode): infinito (infinity) $\infty$ ou paisagem (landscape) $\mathbf{M}$. Não utilizar macro $\boldsymbol{U}$, ou automático $\mathbf{a}$

3. Abertura(aperture): utilizar altos valores def, a fim de maximizar a profundidade de campo.

4. Flash: desligado (2)

5. Balanço de branco (white balance): deve sempre ser ajustado, pois as fontes de luz variam, podendo alterar a cor da foto.

6. Luminosidade: ajuste o foco de luz dos iluminadores de acordo com suas necessidades ou estrutura que deseja fotografar. Lembrando que o copo plástico difunde a luz e reduz o brilho.

7. ISO (International Standards Organization): recomenda-se fotografar com luminosidade adequada e baixos valores de ISO (100 a 400).

8. Qualidade daimagem (image size): idem ao visto para câmeras digitais SLR.

9. Não esquecer o uso de papel milimetrado ou outro tipo de escala próximo ao espécime. 


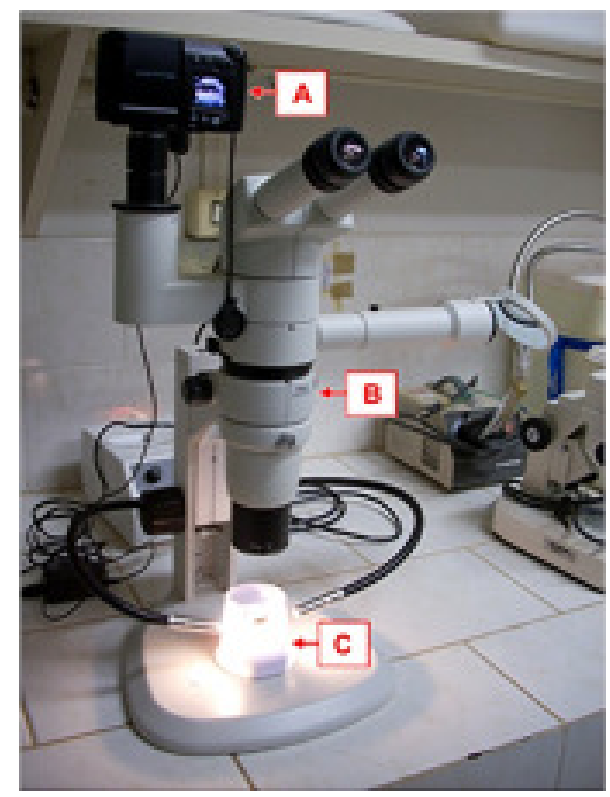

Fig. 2. A. Câmera Nikon ${ }^{o}$, modelo Coolpix 4500. B. Acoplada ao microscópio estereoscópico Nikon $^{\text {ò }}$, modelo SMZ 1000. C. Copo plástico para difusão da luminosidade.

No geral, considerando que o corpo dos insetos não apresenta plano bidimensional, uma única imagem não foi suficiente para mostrar todos os detalhes com nitidez. Assim, foram obtidas fotografias em diferentes distâncias focais, sendo posteriormente otimizadas por meio do software Combine $\mathrm{Z5}^{\mathrm{O}}$, de autoria de Alan Hadley (disponível em: $<$ www.hadleyweb.pwp.blueyonder.co.uk>). O Combine Z5 ${ }^{\circ}$ realiza tarefas semelhantes ao Syncroscopy Auto Montage ${ }^{\circledR}$, com a vantagem de ser um software de domínio público (gratuito). Este software remove todos os elementos de cada fotografia que não estão em foco, combinando as partes que estão em uma única imagem.

O número necessário de fotografias para a obtenção de uma imagem de qualidade variou de acordo com o diâmetro do inseto (média de 5 a 25) e dos níveis de profundidade de campo que se desejou obter.

\section{TRATAMENTO DAS IMAGENS}

A resolução das imagens deve ser escolhida de acordo com a sua utilização e de preferência salvas no formato JPEG. Por convenção, foram tratadas por meio do software Corel Photo-Paint ${ }^{\grave{\partial}}$. Para os que preferem o software Adobe Photoshop ${ }^{\grave{d}}$, maiores detalhes em SELTMAnN 
(2004).

As rotinas de tarefas descritas abaixo foram padronizadas para reduzir a demanda de tempo para o tratamento de cada imagem. Para assegurar que não houvesse alterações ou corte nas estruturas dos insetos, todos os procedimentos foram realizados com o aumento (ferramenta Zoom) Q variando entre 200 a $400 \%$.

Os alfinetes entomológicos transfixados no corpo dos insetos foram eliminados (dependendo da viabilidade e do uso da imagem), utilizando a ferramenta Clone $\mathbf{D D}$. O Tamanho da ponta $\square, \succ, \sqrt[60]{ }$ variou de acordo com a área trabalhada, mas por convenção optou-se pela Forma da ponta não sólida

Após a eliminação dos alfinetes, prosseguiu-se com a padronização e limpeza da superfície de fundo (background).

Este foi selecionado com a ferramenta Varinha mágica $\curvearrowright$ no Modo de tolerância variando entre 10 a 15 Normal HSB 10 ㄴ, dependendo da coloração e morfologia dos insetos.

Altos níveis no Modo de tolerância (acima de 15) tendem a selecionar regiões pertinentes ao inseto, recortando estruturas morfológicas importantes como antenas, asas e tarsos. Baixos níveis no Modo de tolerância (abaixo de 10) tendem a selecionar poucos pixels por vez. Para utilizar a ferramenta Varinha mágica seleciona-se o item aditivo + , clicando com o botão esquerdo no background para que este seja selecionado. Essa ferramenta cria uma "máscara" que envolve todo o corpo do inseto, separando-o do background.

Em seguida, por meio da ferramenta Conta gotas 2 , foi selecionada uma tonalidade de cor, aplicando a ferramenta Preenchimento sobre o background para homogeneização do mesmo.

Procedeu-se então com a remoção da "máscara" (Máscara $>$ Remover) e em seguida foram realizados os ajustes de brilho e contraste (Imagem $>$ Ajustar $>$ Brilho-Contraste-Intensidade). No geral, os ajustes de brilho variaram entre -10 a 10 e os de contraste entre 


\section{-5 a 15.}

No entanto, a acuidade é importante para não ocasionar alterações técnicas desnecessárias e subjetivas. Adequações específicas foram realizadas para cada grupo ou inseto amostrado, devido às variações na coloração e morfologia dos mesmos. Para fins didáticos e taxonômicos, foram inseridas escalas às imagens, que auxiliam no processo de reconhecimento e identificação (Fig, 4).

Imagens produzidas pela câmera digital Nikon ${ }^{\grave{a}}$ acoplada ao estereoscópio foram processadas pelo software Combine $\mathrm{Z5}^{\circ}$. Para um mesmo inseto, foram obtidas fotografias em diferentes distâncias focais (média de 5 a 25), consequentemente, apresentando diferentes níveis de foco. Iniciado o software, no menu (File $>$ New), seleciona-se o conjunto de imagens de interesse. Clicando em (Macro $>$ Do Stack) é realizada a combinação destas em uma única imagem. A imagem é salva por meio do comando (File>Save Frame/Picture As) (Figura 3). Cabe ressaltar que o software Combine $\mathrm{Z}^{\circ}$ apresenta ferramentas para ajuste (Stack), podendo ser realizado um tratamento prévio ao uso do Corel PhotoPaint ${ }^{\mathrm{o}}$.
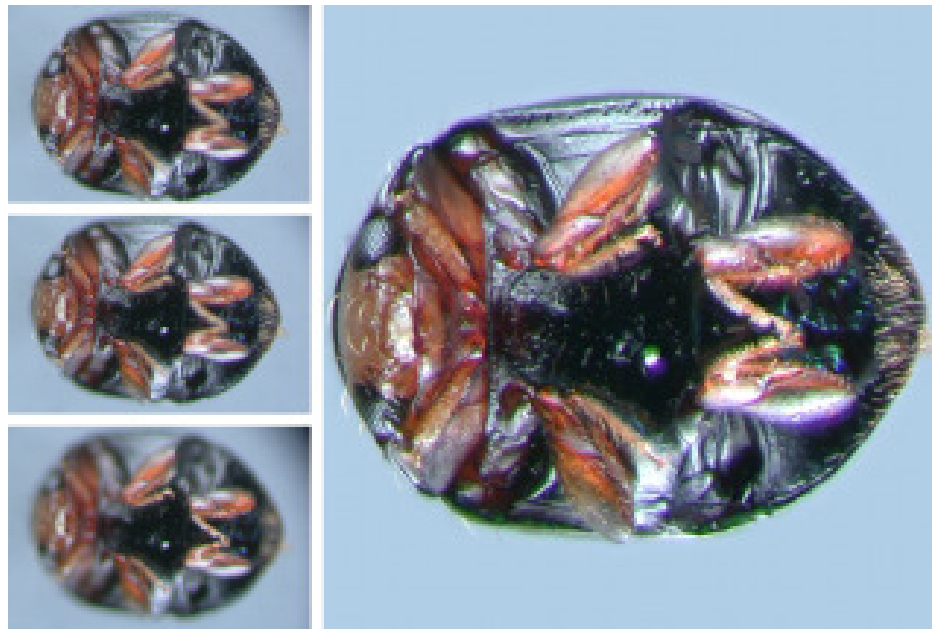

Fig. 3. À esquerda. Imagens obtidas em diferentes distâncias focais. À direita. Imagem final, após combinação pelo software Combine $\mathrm{Z5}^{\circ}$. 
Seguem exemplos de imagens antes e após o tratamento (Figura 4).

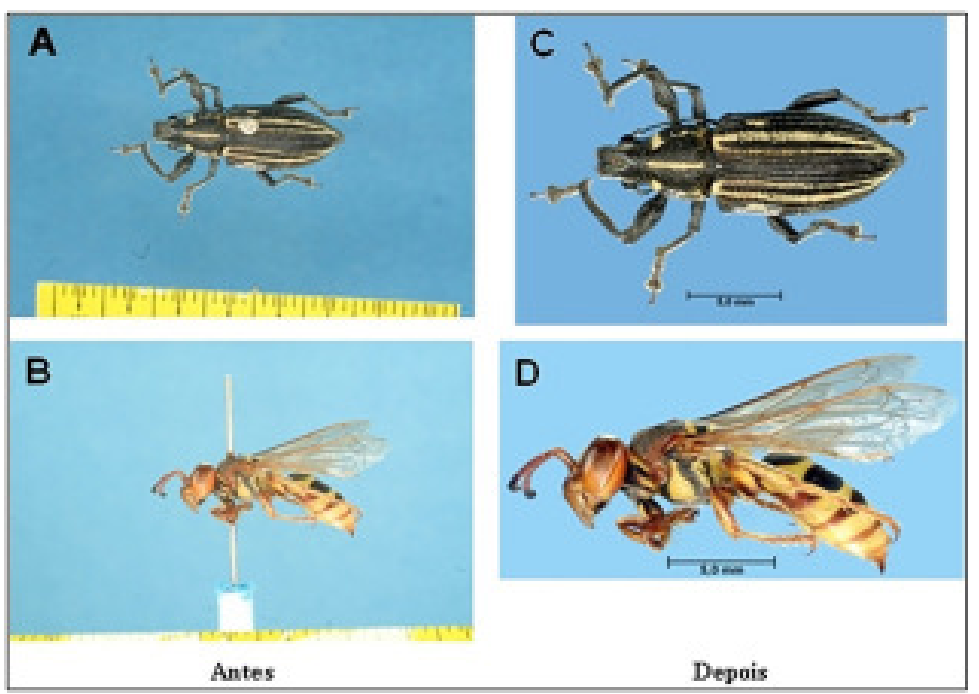

Fig. 4. Imagens antes (A e B) e após (C e D) o processo de tratamento.

\section{RESUMO}

Trabalhos referentes à produção de macrofotografias de insetos são pouco difundidos no meio científico-acadêmico. No entanto, o uso de imagens apresenta vasta aplicação, servindo para publicações científicas, conferências, cursos, banco de dados e na elaboração de chaves de identificação ilustradas. Sendo assim, foram desenvolvidas metodologias que auxiliam na captura e tratamento de imagens em alta resolução, melhorando sua qualidade, sem alterar, no entanto, suas características técnicas.

Palavras-Chave: Entomologia; captura-de-imagens; fotografia.

\section{SUMMARY}

Workings related insects macro-photographs production are little widespread in the scientific community. However, the use of images has wide application, serving for scientific publications, conferences, courses, databases and development of illustrated identification keys. Thus, methodologies were developed to assist in capturing and processing highresolution images, improving its quality, without, however, their technical characteristics.

KEY WORDS: Entomology; images-capture; photograph. 


\section{RÉSUMÉ}

Les travaux sur la production d'insectes macrophotographies petits sont très répandues dans la communauté scientifique. Cependant, l'utilisation des images a une large application, utilisée pour les publications scientifiques, conférences, cours, bases de données et le développement de clés d'identification illustrées. Par conséquent, les méthodes ont été développées pour aider à obtenir des images haute résolution, l'amélioration de sa qualité, sans, toutefois, leurs caractéristiques techniques.

MoTS CLÉs: Entomologie, capture d'image, photographie.

\section{BIBLIOGRAFIA}

Ashworth, S. \& J. Fogarty. A Guide to digitizing insect Collections. Disponível em: <http://insects.oeb.harvard.edu/MCZ/PDFs/ Guide_to_digitizing.pdf $>$. Acesso em 27 ago. 2011.

Azevedo Filho, W.S.; M. Botton \& S.J. Soria. 2007. Curadoria da Coleção Entomológica da. Embrapa. Uva e Vinho. Embrapa Uva e Vinho, Bento Gonçalves. 10 pp. (Comunicado Técnico, 77).

Basset, Y.; V. Novotny; S.E. Miller \& R. Pyle. 2000. Quantifying biodiversity: experience with parataxonomists and digital photography in Papua New Guinea and Guyana. BioScience, 50 (10): 899-908.

Catelli, F.; M. Andreazza; O.G. Júnior \& F.S. da Silva. 2008. Capturando imagens com câmera digital. Física na Escola 9 (2): 3841.

Hochman, B.; F.X. Nahas \& L.M. FerreiRa. 2005. Fotografia aplicada na pesquisa clínico-cirúrgica. Acta Cirúrgica Brasileira 20 (2): 19 25.

KINCH, R.J. Making Digital Camera Microscope Adapters. Disponível em: <http://www.truetex.com/micad.htm>. Acesso em 24 ago. 2011.

PaPAVERo, N. \& U.R. Martins. 1994. Itens da publicação taxonômica. Pp. 69-106. In: PAPAVERo, N. Fundamentos práticos de Taxonomia zoológica (coleções, bibliografia, nomenclatura). (org.). Ed. da Universidade Estadual Paulista, São Paulo. 285 pp.

Seltmann, K. Building web-based interactive keys to the Hymenopteran families and superfamilies. 2004. Lexington, Kentucky, 73 pp. Dissertação (Master of Science in Entomology in the College of Agriculture) - University of Kentucky.

Trevisan, F.; M.A. Scanavini; L.Á. Maltagliati \& R.R. Gonçalves. 2003. Revista Clínica de Ortodontia Dental Press 1 (6): 81-86. 
Walker, A.K. \& T.K. CRosbY. 1988. The preparation and curation of insects. DSIR Science Information Publishing Centre, Wellington. $91 \mathrm{pp}$.

Recebido em 6 de outubro de 2011. 